\section{LA CECEL EN EL CSIC}

\author{
Wifredo Rincón García \\ Representante del CSIC \\ en la Junta de Gobierno de la CECEL
}

\begin{abstract}
The legislative evolution of the Superior Council of Scientist Investigations (CSIC) in connection with the creation and the development of the CECEL and also the connection between the Confederation and the CSIC.
\end{abstract}

KEY WORDS: Spanish Confederation of Centres of Local Studies (CECEL), Superior Council of Scientist Investigations (CSIC).

El día 25 de enero de 1977 el Boletín Oficial del Estado ${ }^{1}$ publicaba un Real Decreto, 62/1977, de 21 de enero, por el que se reestructuraba el Consejo Superior de Investigaciones Científicas, figurando en el Preámbulo -entre los Patronatos que en aquel momento integraban el CSIC- el "José María Quadrado"2. En este mismo Preámbulo, se ponían de manifiesto las consideraciones que hacian necesaria esta reestructuración de la institución: "La necesidad de aprovechar al máximo el potencial de investigación y los recursos existentes para atender selectivamente a los problemas que plantea el desarrollo cultural, científico, tecnológico y económico de la nación, así como la experiencia obtenida en los últimos años sobre el funcionamiento de este complejo sistema, aconsejan una nueva ordenación del Consejo Superior de Investigaciones Científicas que, sobre la base de la ordenación unitaria con la que fue planteado, facilite una mayor coordinación entre los centros de investigación, permita un tratamiento multidisciplinario de los problemas, imponga una simplificación de las estructuras y procedimientos administrativos y favorezca, en fin, una política científica coherente y orientada prioritariamente hacia aquellos objetivos que se juzguen de mayor interés en cada momento".

En el articulado de la Real Orden se dispone en el artículo segundo: "Se suprimen los Patronatos 'Menéndez y Pelayo', 'Raimundo Lulio', 'Alfonso el Sabio', 'Diego Saavedra y Fajardo', 'Juan de la Cierva', 'José María Cuadrado', 'Alonso Herrera' y 'Santiago Ramón y Cajal', siendo sus competencias asumidas por la Junta de Gobierno del Consejo Superior de Investigaciones Científicas, que asume también las

\section{THE CECEL IN THE CSIC}

RESUMEN: Se analiza la evolución legislativa del Consejo Superior de Investigaciones Científicas (CSIC) en relación con el nacimiento y desarrollo de la CECEL, y la relación de la Confederación y el CSIC.

PALABRAS CLAVE: Confederación Española de Centros de Estudios Locales (CECEL). Consejo Superior de Investigaciones Científicas (CSIC).

funciones de su Consejo Ejecutivo, que queda igualmente suprimido".

Llegaba así a su final, con la desaparición del Patronato "José María Quadrado" de Estudios Locales, una época de esplendor de la historiografía local patrocinada por el Consejo Superior de Investigaciones Científicas a través de una red de institutos diseminados por toda España.

Sin embargo, la desaparición de los estudios locales en el seno del Consejo no parecía ser uno de los propósitos de los responsables de la institución, pues en el nuevo Reglamento Orgánico del Consejo Superior de Investigaciones Científicas (Real Decreto 3450/1977, de 30 de diciembre, publicado en el BOE n. ${ }^{\circ}$ 19, de 23 de enero de 1978), en el artículo segundo, en el que se explicitan las "funciones" del Consejo, aparece: "D) Promover la investigación científica en los ámbitos regionales locales, estableciendo, a estos efectos, los oportunos conciertos con las corporaciones y entidades correspondientes".

\section{La creación de la CECEL en el seno del CSIC}

En la Memoria del CSIC correspondiente al año 1977, publicada como es natural en el año siguiente, se hace constar que "1977 fue el año de la unificación del CSIC. El Real Decreto de 21 de enero de 1977, declaró extinguidos los Organismos Autónomos 'Patronato Juan de la Cierva' y 'División de Ciencias Matemáticas, Médicas y 
de la Naturaleza', integrando paralelamente en el CSIC a todo su personal, Centros y Servicios"3, pero nada se dice a propósito de la desaparición de los ocho Patronatos que, órganos intermedios de gobierno, lo integraban, entre ellos el "José María Quadrado".

Sin embargo, y a pesar de la desaparición del "Patronato José María Quadrado", sí que se relacionan en esta Memoria de 1977, en el Directorio de Institutos, Centros y Delegaciones del CSIC por Áreas Temáticas, en el ámbito de la Ciencias del Hombre, los Centros de "Estudios Locales", en cuatro bloques. Por su interés, y por corresponder esta relación al año de desaparición del Patronato, nos parece interesante transcribirlos. En el apartado I, figuran los siguientes Centros subvencionados: en Galicia, el Museo de Pontevedra. En Asturias, el Instituto de Estudios Asturianos, en Oviedo. En el Reino de León, la Institución "Fray Bernardino de Sahagún", en León y el Centro de Estudios e Investigaciones "San Isidoro", también en la capital leonesa; el Centro de Estudios Salmantinos, en Salamanca; la Institución-Casa Museo de Zorrilla, en Valladolid y la Institución "Tello Téllez de Meneses" en Palencia. En Castilla La Vieja, la Institución Cultural "Cantabria", en Santander; la Institución "Fernán González", en Burgos; el Instituto de Estudios Riojanos, en Logroño; el Centro de Estudios Sorianos, en Soria; el Instituto "Diego de Colmenares" en Segovia y la Institución "Gran Duque de Alba", en Ávila. En el apartado II, figuran: en el País Vasco, la Real Sociedad Vascongada de Amigos del País, en San Sebastián; el Grupo "Aranzadi" de la Real Sociedad Vascongada de Amigos del Pais, también en San Sebastián; la Junta Cultural de Vizcaya, en Bilbao y el Consejo de Cultura de la Diputación Foral de Álava, en Vitoria. En Navarra, la Institución "Príncipe de Viana" en Pamplona. En Aragón, el Instituto de Estudios Oscenses, en Huesca; la Institución "Fernando el Católico", en Zaragoza y el Instituto de Estudios Turolenses, en Teruel. En Cataluña, el Instituto de Estudios Ilerdenses, en Lérida; el Instituto de Estudios Gerundenses, en Gerona y el Instituto de Estudios Tarraconenses "Ramón Berenguer IV" de Tarragona. Por último, en las Islas Baleares, el Instituto de Estudios Baleáricos, en Palma de Mallorca; la Sociedad Arqueológica Luliana, también en Palma de Mallorca y el Instituto de Estudios Ibicencos, en Ibiza. En el apartado III, se relacionan los siguientes centros: en Valencia, la Institución "Alfonso el Magnánimo", en Valencia y el Centro de Cultura Valenciana en la misma ciudad; la Sociedad CasteIlonense de Cultura, en Castellón de la Plana y el Instituto de Estudios Alicantinos, en Alicante. En Murcia, la Academia de "Alfonso X el Sabio", en Murcia. En Extremadura, el Instituto de Estudios Extremeños, en Badajoz. En Castilla La Nueva, el Instituto de Estudios Madrileños, en Madrid; la Institución "Marqués de Santillana", en Guadalajara; el Instituto de Estudios Toledanos, en Toledo; el Instituto de Estudios Visigóticos y Mozárabes, en Toledo y el Instituto de Estudios Manchegos, en Ciudad Real. El en apartado IV, el último, se relacionan los siguientes Centros: en Andalucía, el Instituto de Estudios Onubenses "Padre Marchena", en Huelva; el Instituto de Estudios Gaditanos, en Cádiz; el Instituto de Estudios Históricos Jerezanos, en Jerez de la Frontera; el Instituto de Estudios Sevillanos "Rey Alfonso X el Sabio", en Sevilla; la Academia de Bellas Artes "Santa Isabel de Hungria", también en Sevilla; el Instituto de Estudios Malagueños, en Málaga; el Instituto de Estudios Granadinos, en Granada; la Real Academia de Ciencias, Bellas Letras y Nobles Artes, en Córdoba; el Instituto de Estudios Giennenses, en Jaén y el Museo Arqueológico de Linares, en Linares. En Ceuta, el Instituto de Estudios Ceutíes, en Ceuta. En Melilla, no figura ningún centro. Por último, en las Islas Canarias, el Instituto de Estudios Canarios, en La Laguna y el Museo Canario, en las Palmas de Gran Canaria. En total, 51 centros subvencionados, a los que se unian dos propios: el Instituto de Estudios Gallegos "P. Sarmiento", en Santiago de Compostela y el Instituto de Estudios Pirenaicos, en Jaca ${ }^{4}$.

Esta misma relación de centros figuran en las Memorias del CSIC de los años 1978, 1979 y 1980, año este último en el que tuvo lugar en Almagro la celebración de la Asamblea Fundacional de la CECEL.

En la primavera de 1980, el profesor Emilio Sáez, vicepresidente del CSIC y miembro de la Academia de "Alfonso $X$ el Sabio" de Murcia dio el primer paso para la constitución de la Confederación Española de Estudios Locales convocando una reunión que se celebró en Madrid, en la sede del Consejo Superior de Investigaciones Científicas. Disuelto tres años antes el Patronato "José María Quadrado", manifestó Sáez en esta reunión su inquietud y preocupación ante la posible dispersión de estos Centros de Estudios Locales al desvincularse del CSIC, pudiendo desaparecer, tras muchos años, las importantes y fructiferas relaciones establecidas entre ellos. Esta preocupación era también compartida por otros investigadores vinculados a los Centros de Estudios Locales, entre ellos Manuel 
Espadas Burgos, investigador del CSIC y vicedirector del Instituto de Estudios Manchegos.

A pesar de que la asistencia a esta reunión no fue muy numerosa, en esta primera toma de contacto se puso de manifiesto la necesidad de mantener entre los centros un contacto que permitiera el intercambio de sus publicaciones y la colaboración científica y cultural, participando activamente en esta defensa, entre otros representantes de centros, el doctor Adrián Espi, del Instituto de Estudios Alicantinos.

El Instituto de Estudios Manchegos recogió el compromiso y sus representantes en aquella reunión, el mencionado vicedirector doctor Espadas Burgos y la vocal de la Junta de Gobierno, doctora Ángela Madrid Medina, ofrecieron la sede del Instituto para la celebración de un encuentro -que resultaria trascendental- en el que se profundizara en la necesidad de mantener las relaciones entre los centros y sentar las bases del modelo a establecer para su desarrollo. Así, entre los días 16 y 19 de octubre de 1980 se celebró en Ciudad Real y Almagro la XXVII Asamblea de Centros de Estudios Locales, constitutiva de la Confederación Española de Centros de Estudios Locales (CECEL).

El CSIC, estuvo representado por su presidente, don Alejandro Nieto González -quien con su presencia y firma en el acta de constitución de la CECEL prestó un decisivo apoyo al nacimiento de la nueva institución- y su vicepresidente don Emilio Sáez Sánchez. Participaron los siguientes Centros de Estudios Locales: Instituto de Estudios Albacetenses, de Albacete; Instituto de Estudios Alicantinos, de Alicante; Institución "Gran Duque de Alba", de Ávila; Institución "Pedro de Valencia", de Badajoz; Instituto de Estudios Extremeños, de Badajoz; Facultad de Filología de Barcelona; Centro de Estudios Borjanos, de Borja (Zaragoza); Institución "Fernán González", de Burgos; Instituto de Estudios Ceuties, de Ceuta; Instituto de Estudios Manchegos, de Ciudad Real; Real Academia de Ciencias, Bellas Letras y Nobles Artes, de Córdoba; Instituto de Estudios Gerundenses, de Gerona; Instituto de Estudios Altgoaragoneses, de Huesca; Instituto de Estudios Ibicencos, de Ibiza; Instituto de Estudios Gienneses, de Jaén; Centro de Estudios e Investigación "San Isidoro", de León; Instituto de Estudios Madrileños, de Madrid; Instituto "Miguel de Cervantes", de Madrid; Academia de "Alfonso X el Sabio", de Murcia; Instituto de Estudios Asturianos, de Oviedo; Pa- tronato Museo de Pontevedra, de Pontevedra; Instituto de Estudios Salmantinos, de Salamanca; Institución Cultural de Cantabria, de Santander; Centro de Estudios Sorianos, de Soria; Instituto de Estudios Canarios, de La Laguna, Tenerife; Instituto de Estudios Turolenses, de Teruel; Instituto Provincial de Investigación y Estudios Toledanos, de Toledo; Academia de Cultura Valenciana, de Valencia; Institución "Alfonso el Magnánimo", de Valencia e Institución "Fernando el Católico", de Zaragoza. También manifestaron su adhesión otros centros, que no enviaron representación, además del ministro de Investigación y Universidades, don Luis González Seara.

La sesión inaugural de la Asamblea tuvo lugar el día 16 en el Salón de plenos de la Diputación Provincial de Ciudad Real, interviniendo en primer lugar el director del Instituto de Estudios Manchegos, quien afirmó que se iba a constituir la nueva institución cultural a través del universalismo manchego afirmando la realidad de la autonomía local.

Le correspondió entonces intervenir a don José Simón Diaz, quien tras agradecer la cordialidad y hospitalidad que se les había brindado, trazó un breve pero preciso resumen de la vida de los Institutos de Estudios Locales, durante el período 1940-1977, destacando el desinterés y entusiasmo con que más de 2.000 científicos habian venido investigando los distintos ámbitos de la cultura local y señaló que si el anterior período de la vida de dichas Instituciones se podía poner en paralelo con la primera salida de Don Quijote, tras un período de desamparo oficial se iniciaba ahora la segunda con la reación de la Confederación Española de Centros de Estudios Locales integrados en el CSIC.

Con motivo de la asamblea fue inaugurada, en el patio del Palacio Provincial, una interesante exposición bibliográfica en la que se mostraron cerca de trescientas obras, correspondientes tan sólo a diecisiete de los cuarenta y siete centros existentes en esas fechas.

Tras la sesión inaugural, en sesión plenaria y bajo la presidencia de Simón Díaz, comenzó el estudio del proyecto de Estatutos, que constaba de doce artículos, formulándose diversas observaciones. La sesión continuó por la tarde en Valdepeñas y el sábado 18 en el Parador de Turismo de Almagro, finalizándose el estudio del borrador de Estatutos, acordándose su remisión al CSIC para su aprobación por parte de la Institución.

ARBOR CLXXXIV Anejo 1 [2008] 11-18 ISSN: 0210-1963

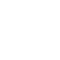


En todas estas sesiones de trabajo se puso de manifiesto el deseo de todos los representantes de los Centros de mantenerse en el seno del CSIC, marco que se consideraba el más apropiado para el desarrollo de labores de investigación de todos los Centros, que, por otra parte, quedaba avalada legalmente, tal como expuso Simón Díaz, por los ya mencionados Real Decreto de 21 de enero de 1977 y el Reglamento Orgánico del Consejo Superior de Investigaciones Cientificas, de 30 de diciembre de 1977, en el que, recordemos, en el apartado "D", del artículo segundo, en el que se ponían de manifiesto las "funciones" del Consejo figura: "Promover la investigación científica en los ámbitos regionales locales, estableciendo, a estos efectos, los oportunos conciertos con las corporaciones y entidades correspondientes".

En el acto de clausura de esta XXVII Asamblea de los Centros de Estudios Locales, fundacional de la CECEL celebrado el domingo 19 de octubre intervino don Manuel Espadas Burgos, vicedirector del Instituto de Estudios Manchegos $y$, como ya hemos apuntado, uno de los principales promotores de las iniciativas que habian culminado con la constitución de la CECEL, congratulándose por el interés demostrado por el ministro González Seara sobre el futuro de los Centros Locales, señalando que "desde ahora van a estrechar sus lazos de relación mediante la labor coordinadora que realizará la Confederación desde el CSIC". José Simón Díaz aprovechó su intervención para aclarar algunas manifestaciones suyas recogidas en prensa, afirmando que "como miembro del CSIC, yo admiro lo realizado por todos sus patronatos que ha sido mucho en los últimos años". Lo que sí dije y reafirmo es que examinando de manera proporcional los recursos de que habían dispuesto cada uno, no llegaba ni al uno por mil los del extinguido Patronato "José María Quadrado". También destacó la labor investigadora de los Centros de Estudios Locales "que dedican un $95 \%$ de sus posibilidades económicas a investigación y una mínima cantidad a gastos de mantenimiento y ello porque sus 2.000 investigadores realizan su trabajo de forma desinteresada" y señaló que con la creación de la CECEL se iniciaba una especie de "segunda salida" de Don Quijote en una etapa de mayor colaboración recíproca con el Consejo, "superada la situación de 'ejército disperso' y el período de aislamiento en que hemos vivido últimamente".
El broche de oro lo puso el presidente del Consejo Superior de Investigaciones Científicas, profesor don Alejandro Nieto González, en el que aludió al papel de cenicienta que han padecido los Estudios Locales, y exaltó las constantes de modestia y fidelidad de los Centros hacia el CSIC, manifestando: "Es momento de olvidar la lamentable historia de las relaciones del CSIC con los Centro de Estudios Locales y proyectarse con ilusión compartida al fututo, actitud esta mía que es asumida por el ministro de Investigación y Universidades. El Consejo está deseando ratificar la permanencia de aquéllos en su seno, cuestión que creo ha quedado consagrada en esta reunión plenaria... No podemos ofrecerle una mano poderosa -añadió- pero sí una relación más sutil y familiar, como parte integrante de la familia de la investigación y de su Consejo Superior..."

Destacó el profesor Nieto que la fórmula de Confederación "era muy apropiada y conforme con la legislación aplicable, organismo que debe basarse más que en criterios de territorialidad, en temática de investigación y anunció que en breves fechas dispondria la CECEL de unos locales en la sede del CSIC, en Madrid, así como de los elementos personales y materiales necesarios 'que ruego me los acepten' manifestó". Estas palabras del Presidente del CSIC fueron acogidas con esperanza y satisfacción por los representantes del medio centenar de Centros asistentes a la Asamblea.

La gestación de la CECEL, que acogía en su seno a los centros que antes integraban el extinto Patronato "José María Quadrado", corrió a cargo de una comisión integrada por representantes de distintos Centros y del CSIC: José Simón Díaz (Instituto de Estudios Madrileños), Manuel Espadas Burgos (CSIC), José Antonio Pérez Rioja (Centro de Estudios Sorianos), Rogelio Pérez Bustamante (Institución Cultural de Cantabria, de Santander) y Martín Almagro Basch (Instituto de Estudios Turolenses) que celebraron varias sesiones de trabajo en Madrid. Otra comisión, integrada en el Instituto de Estudios Manchegos, tuvo a su cargo los trabajos de organización de la Asamblea y tomaron parte en ella José Simón Díaz (presidente), Manuel Espadas Burgos (vicepresidente), Pedro Peral Martín (secretario ejecutivo) y los vocales Ángela Madrid Medina, Ramón José Maldonado y Cocat y José González Lara. La tareas de secretaría fueron desempeñadas por Jorge Sánchez Lillo y Juan Nieva Expósito. 


\section{LA CECEL y EL CSIC}

Presentado al CSIC el borrador de los Estatutos de la CECEL, en la Junta de Gobierno celebrada el día 2 de diciembre del mismo año 1980 se hace constar que: "después de considerar las modificaciones al anteproyecto de Estatuto de la Confederación Española de Centros de Estudios Locales, introducidas por las Comisiones Científica y Económica, las hace suyas y aprueba dicho anteproyecto, que habrá de ser redactado de nuevo teniendo en cuenta las antedichas modificaciones en los artículos 8,11 a), 12 y 13 , y que son las siguientes: $1 .{ }^{a}$ Añadir en el art. 8 la siguiente frase: Formará parte de la Junta de Gobierno, un Vocal representante del CSIC designado por

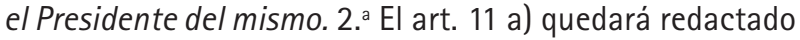
en los siguientes términos: Por las partidas que correspondan dentro del Presupuesto General del CSIC, las cantidades asignadas a los gastos generales de la Agrupación serán independientes de las subvenciones que se asignen individualmente a cada Centro, previa propuesta realizada a tal efecto, de acuerdo con lo prevenido en el art. 10 a). 3. ${ }^{a} \mathrm{El}$ art. 12 en el que se substituye parte integrante del mismo Consejo por Centros vinculados. 4. ${ }^{a}$ Para el ingreso en la Confederación será preciso en todo caso -es decir, incluso en los supuestos del art. 13-, que cada Centro lo solicite individualmente, acreditando que, en el caso de que dependa o esté vinculado con un Organismo Público, cuente con la debida autorización del mismo".

Comunicado este acuerdo de la Junta de Gobierno del CSIC a la CECEL, fue modificado el borrador de Estatutos que, una vez aprobado, fue enviado para su definitiva aprobación gubernativa.

De su articulado destacamos algunos aspectos que nos parecen interesantes, pues ponen claramente de manifiesto las estrechas relaciones entre el CSIC y la CECEL: "La CECEL, encuadrada en el CSIC, agrupa y coordina..." (art. 1. $)^{\circ}$; destaca entre sus cometidos el de "Coordinar dichos Centros en el seno del CSIC" [art. 2..$^{\circ}$ b)]; "Promover las relaciones entre los Centros integrantes y las de éstos con los demás del CSIC" [art. 2..$^{\circ}$ d)]; "Facilitar la colaboración entre los investigadores de una misma especialidad de los distintos Centros y la de éstos en los sectores correspondientes del CSIC" [art. 2. e)]; "La Confederación tendrá su sede en Madrid, en los locales que a tal efecto le asigne el CSIC" (art. 3.); "Resolver los asuntos que le sean encomendados por los órganos del CSIC" [art. 9. $\left.{ }^{\circ} \mathrm{C}\right)$; "Formarán parte de la CECEL,..., los siguientes Centros, por orden cronológico de su vinculación al CSIC" (art. 13.0) y "En caso de disolución, los fondos y bienes existentes en la CECEL serán entregados al CSIC" (art. 15. ).

Poco después de la fundación de la CECEL, el CSIC puso a su disposición dos despachos en la planta cuarta del edificio ubicado en la calle Duque de Medinaceli, 6, de Madrid, además de una administrativa para desempeñar los trabajos diarios bajo las indicaciones de la Presidencia y la Secretaría de la CECEL. En la actualidad, este puesto de administrativo ha sido cubierto desde la CECEL, corriendo a su cargo el correspondiente pago de la persona que lo desempeña.

En el otoño de 2007, cuando los Institutos de Humanidades del CSIC se trasladaron a la nueva sede del Centro de Humanidades y Ciencias Sociales, en la calle Albasanz, la CECEL trasladó igualmente su sede administrativa a este mismo edificio, donde dispone de un despacho para la Presidencia, además de un puesto de trabajo para el administrativo, y donde sigue celebrando sus Juntas de Gobierno trimestrales.

Es importante destacar que, desde la integración de cada uno de los Centros de Estudios Locales en el antiguo Patronato "José María Quadrado" y, posteriormente, en la CECEL, desde la fundación de ésta, el CSIC recibe un ejemplar de cada una de las publicaciones editadas por estos Centros de Estudios Locales, tanto revistas como libros, que pasan a integrar la biblioteca del CSIC, donde están a disposición de toda la comunidad científica, poniéndose así de manifiesto la importancia de las publicaciones llevadas a cabo por lo miembros integrantes de la CECEL, que, sin lugar a dudas, puede considerarse la mayor editorial española, tanto por el número de sus publicaciones, como por la calidad y la variedad temática de las mismas.

Desde su fundación, la CECEL recibe del CSIC una cantidad de dinero con la que hacer frente a sus necesidades organizativas, que se complementa con las cuotas que, desde 2004, abonan todos los centros integrantes de la misma. Las publicaciones de los centros integrantes de la CECEL tienen tradicionalmente un punto de distribución en la Librería Científica del CSIC ubicada en la calle 
Duque de Medinaceli, número 6, de Madrid. El día 12 de septiembre de 2002 fue firmado por la presidenta de la CECEL, doña Ángela Madrid Medina, y el entonces director del Departamento de Publicaciones del CSIC, don Wifredo Rincón García, el convenio que actualmente regula estas ventas.

Mencionaremos también las personas que a lo largo de estos años han desempeñado el cargo de Vocal de la Junta de Gobierno por nombramiento y en representación del CSIC. Éstos han sido, don Manuel Espadas Burgos, don Alberto Sánchez y Álvarez-Insúa y, desde el 26 de septiembre de 2001, don Wifredo Rincón García, investigador científico del CSIC en el Departamento de Historia del Arte -entre septiembre de 2000 y abril de 2005 director del Departamento de Publicaciones del CSIC- y autor de este trabajo.

Para concluir, nos parece interesante destacar el tratamiento que la Confederación de Estudios Locales tuvo en la Memoria del CSIC correspondiente al año 1981. En el "Balance del año 1981", expuesto por el presidente doctor Alejandro Nieto en el salón de actos del edificio central del CSIC, el día 22 de diciembre del mismo año, al referirse a la "reestructuración de Institutos y Centros" y como uno de los últimos logros, se refirió a la "creación de la Confederación Española de Centros de Estudios Locales", que calificó como "merecido acto de justicia para uno de los mejores florones del CSIC, del que tan orgulloso estoy" 5 .

En el Directorio, aparecen por vez primera los Centros de Estudios Locales bajo el epígrafe de "Confederación Española de Centros de Estudios Locales", proporcionándose a lo lectores de la Memoria, una breve información, sobre la misma, extraída de sus Estatutos, que nos parece interesante transcribir: "La Confederación Española de Centros de Estudios Locales encuadrada en el CSIC, agrupa y coordina la actividad la actividad científica de los organismos dedicados a investigación bajo diferentes denominaciones (Centros, Institutos, Academias) y diversas estructuras orgánicas, así como la de aquellos otros Centros que se vayan incorporando en lo sucesivo a ella. La Confederación tiene como atribuciones o finalidades específicas las siguientes: a) Impulsar los estudios de investigaciones locales. b) Coordinar dichos Centros en el seno del CSIC. c) Fomentar y coordinar la creación y desarrollo de los programas genera- les o comunes de investigación. d) Promover las relaciones entre los Centros integrantes y las de éstos con los demás del CSIC, las Universidades y otras Instituciones españolas y extranjeras de finalidad análoga. e) Facilitar la colaboración entre los investigadores de una misma especialidad de los distintos Centros y la de éstos en los sectores correspondientes del CSIC, y los departamentos universitarios afines. f) Promover la labor de los Centros y especialmente de sus producciones bibliográficas y g) Instituir premios y ayudas, organizar reuniones científicas y cuantas otras actividades contribuyan al mejor cumplimiento de sus fines. La Confederación tiene su sede en Madrid, en los locales que a tal efecto le asigna el CSIC"'.

Se relacionan también todos los Centros integrantes de la CECEL, por orden alfabético de localidades. Albacete, Instituto de Estudios Albacetenses; Alicante, Instituto de Estudios Alicantinos; Ávila, Institución "Gran Duque de Alba"; Badajoz, Institución Cultural "Pedro de Valencia" y Centro de Estudios Extremeños; Burgos, Institución "Fernán González"; Cádiz, Academia Provincial de BeIlas Artes; Castellón de la Plana, Sociedad Castellonense de Cultura; Ceuta, Instituto de Estudios Ceuties; Ciudad Real, Instituto de Estudios Manchegos; Córdoba, Real Academia de Ciencias, Bellas Letras y Nobles Artes; Gerona, Instituto de Estudios Gerundenses; Guadalajara, Institución "Marqués de Santillana"; Huesca, Instituto de Estudios Altoaragoneses; Ibiza, Instituto de Estudios Ibicencos; Jaén, Instituto de Estudios Giennenses; Jerez de la Frontera, Centro de Estudios Históricos Jerezanos; La Laguna (Santa Cruz de Tenerife), Instituto de Estudios Canarios; León, Centro de Estudios e Investigación "San Isidoro" e Institución "Fray Bernardino de Sahagún"; Lérida, Instituto de Estudios Ilerdenses; Logroño, Instituto de Estudios Riojanos; Madrid, Instituto de Estudios Madrileños; Murcia, Academia "Alfonso X el Sabio"; Oviedo, Instituto de Estudios Asturianos; Palencia, Institución "Tello Téllez de Meneses"; Palma de Mallorca, Institut d'Estudis Balearics y Sociedad Arqueologico "Lul-liana"; Pontevedra, Museo de Pontevedra; Salamanca, Centro de Estudios Salmantinos; San Sebastián, Real Sociedad Vascongada de Amigos del País; Santander, Institución Cultural "Cantabria" y Centro de Estudios Montañeses; Segovia, Instituto "Diego de Colmenares"; Sevilla, Academia de Bellas Artes "Santa Isabel de Hungría"; Soria, Centro de Estudios Sorianos; Tarragona, Instituto d'Estudis Vallencs, Valls; Teruel, Instituto de Estudios Turolenses; Toledo, 
Instituto de Estudios Toledanos; Valencia, Institución "Alfonso el Magnánimo" y Academia de Cultura Valenciana; Valladolid, Casa Museo de Zorrilla y Zaragoza, Institución "Fernando el Católico"7.

Todos estos Centros se manifiestan como centros subvencionados en el mapa de España que, articulado en Comunidades Autónomas, figura en la misma Memoria ${ }^{8}$.

También se relacionan las publicaciones de los Centros de Estudios Locales, tanto las revistas como los libros, indicándose en el caso de las primeras los números aparecidos en el año y en el caso de los segundos, los títulos y sus correspondientes autores. Igual que sucedía cuando se relacionaban los Centros, a modo de preámbulo de las publicaciones figura este texto que consideramos interesante transcribir, pues se trataba de una precisa información sobre la CECEL y sus actividades, para conocimiento de los investigadores y otro personal del CSIC.

El texto es el siguiente: "El 28 de abril (1981) se verificó en Madrid la constitución oficial de la Confederación Española de Centros de Estudios Locales, para agrupary coordinar la actividad cientifica de los organismos dedicados a la investigación bajo diferentes denominaciones y diversas estructuras orgánicas en los ámbitos regional y local. Para valorar la oportunidad y la importancia de este acto, basta recordar que, pocos días después, los veintiún ministros de Cultura de los paises miembros del Consejo de Europa reunidos en Luxemburgo acordaron promover la conservación y el desarrollo de las culturas regionales y locales y las trascendencia creciente del tema en la nueva configuración del Estado. Aunque el aspecto más conocido de la labor desarrollada es el de las publicaciones, tanto eruditas como divulgadoras, existen otros muchos de no menor interés, como son las excavaciones arqueológicas, restauraciones de monumentos, creación de archivos, bibliotecas y museos, congresos científicos, concesiones de premios y becas, etc. Cada centro suele tener una publicación periódica, aunque son varios los que mantienen varias a la vez, como las Instituciones 'Fernando el Católico', de Zaragoza, y 'Alfonso el Magnánimo', de Valencia, pudiendo señalarse también, como indicio de la magnitud de los realizado en cuanto a libros y folletos, que la primera de estas entidades acaba de lanzar su obra número 792. En la ciudad de Badajoz se celebró, durante los primeros días de octubre, la XXVIII Reunión Plenaria de los Centros de Estudios Locales, celebrándose a la vez sesiones de trabajo en que se estudiaron comunicaciones y ponencias sobre el tema Los caminos de la Península lbérica, tratado desde los puntos de vista histórico, geográfico, artístico, social, económico, literario, etc. A la vez, la magna Exposición Bibliográfica mostró la totalidad de la producción editorial de los Centros, que será reseñada en un Catálogo, destinado a testimoniar el alcance de la aportación prestada por estos organismos a la Cultura española"9.

Como podemos ver, un interesante y clarificador texto cuya finalidad era divulgar la actividad de los centros integrados en la CECEL.

Por último, recogemos el texto de la Secretaria de Estado de Universidades e Investigación, doctora doña Carmina Virgili, leído en la sesión de clausura de la XXX Asamblea Plenaria de la CECEL, celebrada en La Laguna, el 2 de octubre de 1983, en el que valoró, según se pone de manifiesto en el Memoria del CSIC de 1983, donde se transcribe, "la aportación de la CECEL dentro del panorama científico español". Su intervención, de gran interés, fue la siguiente: "Los Centros de Estudios Locales del CSIC mantienen la mejor y más difícil de las tradiciones culturales y científicas de nuestro pais. La de los 'caballeritos de Azcoitia', la de las Sociedades de Amigos del Pais, la de los Ateneos, la de los hombres o grupos que aislados de apoyos pero nunca de la realidad de su entorno lucharon, a veces, pagando un alto precio por abrir caminos a la razón y al conocimiento. Pero vuestros Centros no significan únicamente la continuidad de una tradición tantas veces negada y abatida. Son también en más de un sentido parte de un modelo que tenemos que construir. Vuestra actividad interdisciplinar. Vuestros trabajos apegados a la realidad de vuestro entorno. Vuestra capacidad de llevar a la realidad inmediata los conocimientos culturales y científicos. El extraer de esta misma realidad nuevos datos de conocimiento. Todo esto, unido a una descentralización que vosotros mismos habéis impedido que se convierta en dispersión, tiene mucho que ver con la necesaria incorporación de nuestra ciencia y nuestra cultura a la modernidad. $Y$ con sus posibilidades de enriquecer en todos los aspectos el medio social concreto en que la ciencia y la cultura se desarrollan. La flexibilidad, desburocratización y apertura de vuestros Centros no son cualidades que nieguen o impi-

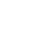


dan su posible y positiva apertura y colaboración con el mundo universitario. Muy al contrario, ofrecen notas que han de ser características de la nueva Universidad -profesionalizada, autónoma, ligada a su entorno social-que queremos empezar a construir y que este mismo curso inicia su proceso de reforma, su andadura autonómica y establece nuevos lazos con las comunidades autónomas en las que están ancladas. Vuestra integración en el CSIC no sólo garantiza la intercomunicación y la unión de esfuerzos, supone también vuestro compromiso con el proyecto de vigorización y renovación al servicio de su sociedad"10.
Recibido: Octubre de 2007

Aceptado: Mayo de 2008

\section{NOTAS}

1 BOE número 21, 1977, pp. 1719-1720.

2 Figura en el texto como "Cuadrado".

3 Memoria del CSIC, 1977, p. 7.

4 Memoria del CSIC, 1977, pp. 371374.
5 Memoria del CSIC, 1981, p. 8.

6 Memoria del CSIC, 1981, p. 251.

7 Memoria del CSIC, 1981, pp. 252254.

8 Memoria del CSIC, 1981, p. 19.

9 Memoria del CSIC, 1981, p. 217.

10 Memoria del CSIC, 1983, p. 225. 